Konrad-Zuse-Zentrum für Informationstechnik Berlin Heilbronner Str. 10, D-10711 Berlin - Wilmersdorf

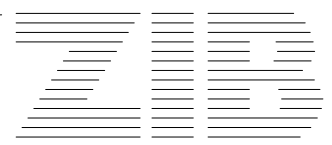

FOLKMAR A. BORNEMANN

\title{
Interpolation Spaces and
} Optimal Multilevel Preconditioners*

* appears in:

Proceedings of the 7th International Conference on Domain Decomposition, D. Keyes and J. Xu (eds.), CONM series of the AMS

SC 93-33 (Dezember 1993) 


\title{
INTERPOLATION SPACES AND OPTIMAL MULTILEVEL PRECONDITIONERS
}

\author{
FOLKMAR A. BORNEMANN
}

\begin{abstract}
This paper throws light on the connection between the optimal condition number estimate for the BPX method and constructive approximation theory. We provide a machinery, which allows to understand the optimality as a consequence of an approximation property and an inverse inequality in $H^{1+\epsilon}, \epsilon>0$. This machinery constructs so-called approximation spaces, which characterize a certain rate of approximation by finite elements and relates them with interpolation spaces, which characterize a certain smoothness.
\end{abstract}

\section{INTRODUCTION}

For simplicity we consider the following elliptic boundary problem of second order on a polygonal domain $\Omega \subset \mathbb{R}^{2}$ :

$$
-\Delta u+u=f,\left.\quad \partial_{n} u\right|_{\partial \Omega}=0,
$$

where $f \in L^{2}(\Omega)$. The weak solution $u \in H^{1}(\Omega)$ is given by the variational problem

$$
a(u, v):=(\nabla u, \nabla v)_{L^{2}}+(u, v)_{L^{2}}=(f, v)_{L^{2}} \quad \forall v \in H^{1}(\Omega),
$$

where we use a suggestive notation of the scalar products in $L^{2}(\Omega)^{2}$ and $L^{2}(\Omega)$. Let $\left(\mathcal{T}_{j}\right)_{j}$ be a sequence of nested regular quasi-uniform triangulations of $\Omega$ with mesh-size parameter

$$
h_{j}:=\max _{T \in \mathcal{T}_{j}} \operatorname{diam}(T) \approx 2^{-j} .
$$

Throughout this paper we use the notation $a \lesssim b$ iff there is a constant $c>0$, such that $a \leq c b$ and $a \approx b$ iff $a \lesssim b$ and $a \gtrsim b$. Those constants $c$ will be independent of everything, with the only exception that they might depend on $\Omega$ and the shape regularity of the triangulations.

Introducing the spaces of linear finite elements

$$
X_{j}=\left\{u \in C(\bar{\Omega}):\left.u\right|_{T} \in P_{1}(T) \quad \forall T \in \mathcal{T}_{j}\right\},
$$

where $P_{1}(T)$ denotes the linear functions on the triangle $T$, we get

$$
X_{0} \subset X_{1} \subset \ldots \subset X_{j} \subset \ldots \subset H^{1}(\Omega) .
$$

1991 Mathematics Subject Classification. Primary 65N55, 65N30; Secondary 41A65, 46B70. 
The finite element operator $A_{j}: X_{j} \rightarrow X_{j}$ defined by

$$
\left(A_{j} u, v_{j}\right)_{L^{2}}=a\left(u, v_{j}\right) \quad \forall v_{j} \in X_{j}
$$

deserves to be preconditioned for the actual efficient computation. Thus we are looking for an easily invertible operator $B_{j}: X_{j} \rightarrow X_{j}$ such that $A_{j} \approx B_{j}$, i.e.,

$$
\left(A_{j} u, u\right)_{L^{2}} \approx\left(B_{j} u, u\right)_{L^{2}} \quad \forall u \in X_{j}
$$

Bramble, Pasciak and $\mathrm{Xu}[3]$ constructed the preconditioner

$$
B_{j}=A_{0} Q_{0}+\sum_{k=1}^{j} 4^{k}\left(Q_{k}-Q_{k-1}\right),
$$

where $Q_{k}: L^{2}(\Omega) \rightarrow X_{k}$ are the $L^{2}$-orthogonal projections. They were originally able to prove

$$
\frac{1}{j+1}\left(B_{j} u, u\right)_{L^{2}} \lesssim\left(A_{j} u, u\right)_{L^{2}} \lesssim(j+1)\left(B_{j} u, u\right)_{L^{2}} \quad \forall u \in X_{j}
$$

Their prove was based on the observation that

$$
\left(A_{k} u, u\right)_{L^{2}} \approx 4^{k}\|u\|_{L^{2}}^{2} \quad \forall u \in\left(Q_{k}-Q_{k-1}\right) L^{2}(\Omega)
$$

which is a fairly easy consequence of the approximation property

$$
\left\|u-Q_{k} u\right\|_{L^{2}} \lesssim h_{k}\|u\|_{H^{1}} \quad \forall u \in H^{1}(\Omega)
$$

and the inverse inequality

$$
\left\|u_{k}\right\|_{H^{1}} \lesssim h_{k}^{-1}\left\|u_{k}\right\|_{L^{2}} \quad \forall u_{k} \in X_{k}
$$

Oswald [10] was the first who observed a strong link of this method of preconditioning to approximation theory, a link, which in fact immediately supplies a prove for the optimal result

$$
A_{j} \approx B_{j}
$$

Since then several authors contributed to that result within one of the following two respects: generalization or construction of more or less elementary proofs $[2,6,7,14$, 15]. The aim of this paper is to clarify the link to approximation theory by making available an easily accessible framework. Moreover it will turn out, that the main ingredients of the proof are inequalities like (1.2) and (1.3). 


\section{Approximation Spaces are Interpolation Spaces}

The optimality result (1.4) would be a straightforward consequence of the following norm equivalence with scaling exponent $\theta=1$ :

$$
\|u\|_{H^{1}}^{2} \approx\|u\|_{L^{2}}^{2}+\sum_{k=0}^{\infty}\left(2^{k \theta} E_{k}(u)\right)^{2} \quad \forall u \in H^{1}(\Omega)
$$

where $E_{k}(u)$ denotes the error of best approximation in $L^{2}(\Omega)$

$$
E_{k}(u)=\inf _{v_{k} \in X_{k}}\left\|u-v_{k}\right\|_{L^{2}}=\left\|u-Q_{k} u\right\|_{L^{2}} .
$$

We now ask the rather abstract question: Which sequences $\left(X_{j}\right)_{j}$ of nested finitedimensional subspaces of $L^{2}(\Omega)$ allow for some scaling exponent $\theta$ such that the norm equivalence (2.1) holds?

Rather than answering this question directly, we define Banach spaces $A^{\theta} \hookrightarrow L^{2}(\Omega)$ by the norms given as the right hand sides of (2.1),

$$
\|u\|_{A^{\theta}}^{2} \approx\|u\|_{L^{2}}^{2}+\sum_{k=0}^{\infty}\left(2^{k \theta} E_{k}(u)\right)^{2} .
$$

These approximation spaces $A^{\theta}$, which measure by $\theta$ how well their elements can be approximated by the spaces $\left(X_{j}\right)_{j}$, were introduced by Peetre $[5,11]$ in the early sixties and have been intensively studied in approximation theory since then. It should be mentioned that the results, which will follow, had been known in a somewhat different form to the Russian school around Nikol'skil as early as the fifties.

Our starting question reads now: Is there a $\theta$ such that $A^{\theta}=H^{1}(\Omega)$ ? But exactly this question is a key issue of approximation theory — it requires the characterization of the approximation spaces through smoothness spaces like the Sobolev spaces. The answer given by Peetre [5, 11] was a relation between the approximation spaces $A^{\theta}$ and the interpolation spaces $\left(L^{2}(\Omega), X\right)_{\sigma, 2}$, where $X$ is some "nice" space with $X_{k} \subset X \subset L^{2}(\Omega)$ for all $k \geq 0$.

Theorem 1. Let $\alpha>0$. An approximation property (Jackson inequality) $J_{\alpha}$, i.e.,

$$
\left\|u-Q_{k} u\right\|_{L^{2}} \lesssim 2^{-k \alpha}\|u\|_{X} \quad \forall k \geq 0, u \in X,
$$

implies the embedding

$$
\left(L^{2}(\Omega), X\right)_{\sigma, 2} \hookrightarrow A^{\sigma \alpha} \quad 0<\sigma<1
$$

Let $\beta>0$. An inverse inequality (Bernstein inequality) $B_{\beta}$, i.e.,

$$
\left\|u_{k}\right\|_{X} \lesssim 2^{k \beta}\left\|u_{k}\right\|_{L^{2}} \quad \forall k \geq 0, u_{k} \in X_{k}
$$


implies the embedding

$$
A^{\sigma \beta} \hookrightarrow\left(L^{2}(\Omega), X\right)_{\sigma, 2} \quad 0<\sigma<1 .
$$

Remark 1. Note that a standard interpolation argument applied to the Jackson inequality $J_{\alpha}$ for $X$ and to the trivial estimate $\left\|u-Q_{k} u\right\|_{L^{2}} \lesssim\|u\|_{L^{2}}$ would only reveal an approximation property for the interpolation spaces

$$
2^{k \alpha \sigma}\left\|u-Q_{k} u\right\|_{L^{2}} \lesssim\|u\|_{\left(L^{2}(\Omega), X\right)_{\sigma, 2}} \quad 0<\sigma<1 .
$$

This result would be considerably weaker than the assertion of Theorem 1 , which states that the right hand sides of (2.4) are in fact square summable as a sequence of $k$.

If we use an appropriate method for the construction of the interpolation spaces $\left(L^{2}(\Omega), X\right)_{\sigma, 2}$, the proof of Theorem 1 is utmost simple. We demonstrate this for the first part concerning the Jackson inequality.

Proof. Fix some $0<\lambda<1$. Using the discrete version of Peetre's K-method of interpolation $[1,5,13]$ we get

$$
\|u\|_{\left(L^{2}(\Omega), X\right)_{\sigma, 2}}^{2}=\sum_{k \in \mathbb{Z}}\left(\lambda^{-k \sigma} K\left(\lambda^{k}, u\right)\right)^{2} .
$$

The following estimates relate the $K$-functional with the error of best approximation by using the Jackson inequality $J_{\alpha}$ : For all $k \geq 0$

$$
\begin{aligned}
E_{k}(u) & \leq \inf _{v \in X}\left\|u-Q_{k} v\right\|_{L^{2}} \\
& \leq \inf _{v \in X}\left(\|u-v\|_{L^{2}}+\left\|v-Q_{k} v\right\|_{L^{2}}\right) \\
& \lesssim \inf _{v \in X}\left(\|u-v\|_{L^{2}}+2^{-k \alpha}\|v\|_{X}\right)=: K\left(2^{-k \alpha}, u\right) .
\end{aligned}
$$

Thus, by making the choice $\lambda=2^{-\alpha}$, we end up with

$$
\|u\|_{\left(L^{2}(\Omega), X\right)_{\sigma, 2}}^{2} \gtrsim \sum_{k=0}^{\infty}\left(2^{k \sigma \alpha} E_{k}(u)\right)^{2}+\|u\|_{L^{2}}^{2}=\|u\|_{A^{\sigma \alpha}}^{2} .
$$

Let us note, that the discrete version of Peetre's J-method of interpolation $[1,5,13]$ turns out to be appropriate for the proof of the second part of the Theorem.

Corollary 1. The approximation property is restricted by the inverse inequality, i.e., $J_{\alpha}$ and $B_{\beta}$ imply $\alpha \leq \beta$. For $\alpha=\beta$ we get the identification

$$
A^{\sigma \alpha}=\left(L^{2}(\Omega), X\right)_{\sigma, 2}, \quad 0<\sigma<1 .
$$


Proof. If $J_{\alpha}$ and $B_{\beta}$ hold, Theorem 1 gives $A^{\sigma \beta} \hookrightarrow A^{\sigma \alpha}$ for $0<\sigma<1$. This embedding is equivalent to $\alpha \leq \beta$, as can easily be shown.

\section{Application to Linear Finite Elements}

In order to answer the question from the beginning of our consideration, Corollary 1 leads us to the following strategy: Choose $X$ and $0<\sigma<1$, such that

$$
H^{1}(\Omega)=\left(L^{2}(\Omega), X\right)_{\sigma, 2} .
$$

In any case this requires that $X$ is smoother than $H^{1}(\Omega)$. Interpolation theory in Sobolev spaces $[13,1]$ states for minimal smooth domains $\Omega$ (i.e., $\Omega$ allows a continuous extension operator $E: H^{s}(\Omega) \rightarrow H^{s}\left(\mathbb{R}^{2}\right)$ for all $\left.s \geq 0\right)$, that

$$
H^{1}(\Omega)=\left(L^{2}(\Omega), H^{s}(\Omega)\right)_{1 / s, 2} \quad \forall s>1 .
$$

In our context it suffices to know, that a polygonal domain $\Omega$ without slits is minimal smooth [12]. Now it turns out, that the finite element spaces fulfill

$$
X_{k} \subset H^{s}(\Omega) \quad \Longleftrightarrow \quad 0 \leq s<3 / 2 .
$$

For the following we fix some $1<s=1+\epsilon<3 / 2$ and we can apply Theorem 1 as soon as we have established an approximation property and an inverse inequality in $H^{1+\epsilon}$. We obtain the approximation property $J_{s}$, i.e.,

$$
\left\|u-Q_{k} u\right\|_{L^{2}} \lesssim h_{k}^{s}\|u\|_{H^{s}} \lesssim 2^{-k s}\|u\|_{H^{s}} \quad \forall u \in H^{s}(\Omega),
$$

by simple interpolation between the situations $s=0$ and $s=2$ as indicated in Remark 1. A little bit deeper lies the inverse inequality $B_{s}$, i.e.,

$$
\left\|u_{k}\right\|_{H^{s}} \lesssim h_{k}^{-s}\left\|u_{k}\right\|_{L^{2}} \lesssim 2^{k s}\left\|u_{k}\right\|_{L^{2}} \quad \forall u_{k} \in X_{k}
$$

which can be proved using the Sobolev-Slobodeckiu norm

$$
\|u\|_{H^{1+\epsilon}}^{2} \approx\|u\|_{H^{1}}^{2}+\int_{\Omega} \int_{\Omega} \frac{|\nabla u(x)-\nabla u(y)|^{2}}{|x-y|^{2+2 \epsilon}} d x d y
$$

of $H^{s}(\Omega)$, cf. [4]. Thus we have $\alpha=\beta=1 / \sigma$ and Corollary 1 states the equivalence, which makes the BPX preconditioner optimal:

Theorem 2. For linear finite elements the equivalence $A^{1}=H^{1}(\Omega)$ holds.

Remark 2. This Theorem and the more general equivalence "approximation space = smoothness space", i.e, $A^{s}=H^{s}(\Omega)$ for $s$ from some interval, holds generically for a lot of sequences $\left(X_{j}\right)_{j}$, like higher order finite elements, spectral methods and wavelets. Details can be found in $[8,9]$. 
Our considerations show, that it is reasonable to view the approximation property (3.1) and the inverse inequality (3.2) in $H^{1+\epsilon}(\Omega), \epsilon>0$ arbitrary small, as the "chief cause" for the optimality of BPX. In the original proof of the weaker result (1.1) the corresponding estimates in $H^{1}(\Omega)$ have been the working ground. Thus, the essential step is, to use the fact that linear finite elements are a little bit smoother than one usually thinks. This essential step is hidden in one way or another in all proofs $[2,6,7,10,14,15]$ of the optimality of the BPX preconditioner.

\section{REFERENCES}

1. J. Bergh and J. Löfström, Interpolation Spaces. An Introduction, Springer-Verlag, Berlin, Heidelberg, New York, 1976.

2. J. H. Bramble and J. E. Pasciak, New estimates for multilevel algorithms including the V-cycle, Math. Comp. 60 (1993), 447-471.

3. J. H. Bramble, J. E. Pasciak, and J. Xu, Parallel multilevel preconditioners, Math. Comp. 55 (1990), 1-22.

4. quadratic forms, Math. Comp. 56 (1991), 1-34.

5. P. L. Butzer and K. Scherer, Approximationsprozesse und Interpolationsmethoden, Bibliographisches Institut, Mannheim, 1968.

6. F. A. Bornemann and $\mathrm{H}$. Yserentant, A basic norm equivalence for the theory of multilevel methods, Numer. Math. 64 (1993), 455-476.

7. W. Dahmen and A. Kunoth, Multilevel preconditioning, Numer. Math. 63 (1992), 315-344.

8. R. DeVore and G. G. Lorentz, Constructive Approximation. I, Springer-Verlag, Berlin, Heidelberg, New York, 1993.

9. P. Oswald, On function spaces related to finite element approximation theory, Z. Anal. Anwend. 9 (1990), 43-64

10. P. Oswald, On discrete norm estimates related to multilevel preconditioners in the finite element method, Proc. Int. Conf. Constructive Theory of Functions, Varna, 1991.

11. J. Peetre, A Theory of Interpolation in Normed Spaces, Notes Universidade de Brasilia, 1963.

12. E. M. Stein, Singular Integrals and Differentiability Properties of Functions, Princeton University Press, Princeton, 1970.

13. H. Triebel, Interpolation Theory, Function Spaces, Differential Operators, North-Holland Pub. Comp., Amsterdam, New York, Oxford, 1978.

14. J. Xu, Iterative methods by space decomposition and subspace correction, SIAM Review 34 (1992), 581-613.

15. X. Zhang, Multilevel Schwarz methods, Numer. Math. 63 (1992), 521-539.

Fachbereich Mathematik, Freie Universität Berlin, Germany

Current address: Konrad-Zuse-Zentrum Berlin, Heilbronner Str. 10, 10711 Berlin, Germany

E-mail address: bornemann@sc.zib-berlin.de 